\title{
La responsabilidad social como estrategia de protección social en el capitalismo contemporáneo
}

\author{
Mariangel Sánchez Alvarado \\ https://orcid.org/0000-0001-8527-119X
}

\author{
Tânia Maria Santana dos Santos ${ }^{2}$ \\ https://orcid.org/0000-0001-8558-8656
}

${ }^{1}$ Universidade Federal de Alagoas, Departamento de Serviço Social, Programa de Pós-Graduação em Serviço Social, Maceió, AL, Brasil

${ }^{2}$ Universidade Federal de Mato Grosso, Departamento de Serviço Social, Cuiabá, MT, Brasil

\section{La responsabilidad social como estrategia de protección social en el capitalismo contemporáneo}

Resumen: Se presentan los elementos históricos y contextuales que posibilitaron el surgimiento de la Responsabilidad Social a partir de un análisis crítico, partiendo de la totalidad social para entender el objeto de estudio más allá de su sentido endógeno. Se inicia a partir de la contrarreforma del Estado en los años 1980 causado por las necesidades del modo de producción capitalista para su reproducción y cómo esto se fue implementando en los gobiernos de los países de América Latina por medio de los Planes de Ajuste Estructural, utilizando estrategias para la privatización de servicios sociales como lo fue la Responsabilidad Social, entendida como uno de los factores que potencializa la hegemonía burguesa articulando las acciones empresariales y de organizaciones la sociedad civil a partir del discurso de responsabilidades compartidas, con particularidades político-ideológico y fundamentalmente económicas, por lo cual, es sin duda una forma que potencializa la valorización del capital.

Palabras claves: Responsabilidad social. Estado. Contrarreforma. Neoliberalismo.

\section{A responsabilidade social como estratégia de proteção social no capitalismo contemporâneo}

Resumo: Este artigo apresenta elementos históricos e contextuais que possibilitaram a emergência da responsabilidade social a partir de uma análise crítica, partindo da totalidade social para compreender o objeto de estudo além de seu significado endógeno. Inicia-se a partir da contrarreforma do Estado nos anos 1980, causada pelas necessidades do modo de produção capitalista para sua reprodução, e como foi implementada nos governos dos países latino-americanos através dos Planos de Ajustamento Estrutural utilizando estratégias para a privatização de serviços sociais como o foi a responsabilidade social, entendida como um dos fatores que potencializam a hegemonia burguesa ao articular as ações empresariais e de organizações da sociedade civil a partir do discurso de responsabilidades compartilhas, com particularidades políticoideológicas e fundamentalmente econômicas, razão pela qual é, sem dúvida, uma forma que potencializa a valorização do capital.

Palavras-chave: Responsabilidade social. Estado. Contrarreforma. Neoliberalismo.

\section{Social responsibility as a social protection strategy in modern capitalism}

Abstract: This article presents historical and contextual elements that promoted the emergence of social responsibility, based on critical analysis and on social totality to understand the study object beyond its endogenous meaning. The study starts from the counter-reform of the state in the 1980s - which was caused by the needs of the capitalist mode of production and was carried out for its reproduction - and describes how the counter-reform was implemented in Latin American countries, based on Structural Adjustment Plans. These plans used strategies to privatize social services, such as the notion of social responsibility that increases the bourgeois hegemony by coordinating corporate's and civil society organizations' actions with the rationale of "shared responsibility," supported by politicoideological and economic particularities that enhance the value of capital.

Keywords: Social responsibility. State. Counter-reforms. Neoliberalism.

Recibido en 01.06.2018. Aprobado en 18.09.2018. Revisado en 27.02.2019.

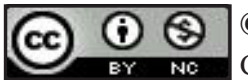

(C) El(Los) Autor(es). 2019 Acceso Abierto Esta obra está licenciada bajo los términos de la Licencia Creative

Commons Atribución-NoComercial 4.0 Internacional (https://creativecommons.org/licenses/by-nc/4.0/deed.es), que permite copiar, distribuir y reproducir en cualquier medio, así como también adaptar, transformar y crear a partir de este material, desde que para fines no comerciales, y que usted fornezca el crédito debido a los autores y a la fuente, insiera un enlace para la Licencia Creative Commons e indique si fueron hechas alteraciones. 


\section{Introducción}

El neoliberalismo es tomado como ideario principal para dar respuesta a la crisis vivida en los años 1980 por medio de los procesos de reestructuración del capital, esto trajo consigo una flexibilización de los mercados nacionales e internacionales, de las relaciones laborales, de producción, de inversión financiera, del distanciamiento del Estado de sus responsabilidades sociales y de la regulación social entre capital y trabajo, estableciéndose, como instrumento de consolidación hegemónica del capital mediante su papel central en el proceso de desregularización y reforma estatal y en la reestructuración productiva.

Una de las características del capitalismo en la fase neoliberal es la desresponsabilización de la actuación del Estado en las manifestaciones de la cuestión social. Como una estrategia para conseguir esto, se promueve un discurso de solidaridad y responsabilidad compartidas entre los diferentes sectores de la población, transfiriendo actividades realizadas históricamente por el Estado para el mercado o para la sociedad civil, promoviendo la refilantropización, remercantilización y despolitización de su actuación.

Además, promulgaba e incentivaba la transferencia de las responsabilidades sociales del Estado para otros sectores. Así se fortaleció el tercer sector, facilitando y aumentando la participación de las empresas en acciones sociales, como el voluntariado, la filantropía, inversiones y convenios con entidades sin fines lucrativos.

Sin embargo, en este sentido, el objetivo de retirar del Estado la responsabilidad de intervención en la cuestión social y de transferirla para la esfera del tercer sector, no ocurre por motivos de eficiencia ni por razones financieras de reducir los costos necesarios para sustentar esta función estatal; el motivo es fundamentalmente político-ideológico, donde se procura retirar y vaciar la dimensión de derecho universal del ciudadano con relación a las políticas sociales de calidad. De esta manera, se busca una cultura de raíz positivista de autoculpabilización de los individuos por las situaciones que viven y por lo tanto, la manera de resolver estos problemas es por medio de la autoayuda y ayuda mutua, exonerando el capital de sus responsabilidades y facilitar una nueva y abundante demanda lucrativa para el sector empresarial.

Es en este contexto que emerge la Responsabilidad Social, con un discurso de responsabilidades compartidas y un direccionamiento de la solución o intervención a las demandas de la cuestión social a partir de la movilización social de la comunidad local, organizaciones no gubernamentales (ONGs), empresas privadas y por medio de los supuestos convenios con el Estado.

Se comprende la Responsabilidad Social no como buena voluntad de los empresarios ni como estrategia interna de las empresas, sino dentro de las relaciones del sistema de producción capitalista, el cual, históricamente, presenta diferentes respuestas para su consolidación y expansión en sus diferentes momentos de crisis.

El panorama para comprender las determinaciones que dieron origen a la Responsabilidad Social debe de tener como referente las respuestas del sistema de producción capitalista que, para mantener su estabilidad y legitimidad, promueve un Estado mínimo. Así, a partir de los años 1990 se comienza a trasladar algunas funciones sociales atribuidas al Estado para el sector privado ${ }^{2}$.

Frente a este escenario, se definió como objeto de estudio la Responsabilidad Social, la cual abarca no solamente la intervención empresarial, también incluye, según la Organización Internacional de Normalización (ISO), la intervención social de las organizaciones de la sociedad civil y de las instituciones del Estado.

En este estudio, es entendida como uno de los factores que potencializa la hegemonía burguesa y como estrategia de rearticulación de las acciones de diferentes sectores por medio de un nuevo consenso burgués, político, ideológico y fundamentalmente económico. El objeto de estudio toma prominencia a partir de la interrogante principal, la cual encaminó el proceso investigativo: ¿Cuál fue la coyuntura histórica que posibilitó el surgimiento de la Responsabilidad Social en el contexto capitalista contemporáneo?

El objetivo general de esta investigación consistió en analizar los condicionantes que influenciaron en el surgimiento de la Responsabilidad Social como protección social privada en el capitalismo contemporáneo. Para conseguir esto, es trascendental analizar el movimiento del capital y como esto influye en el Estado y en la intervención de este en la cuestión social, lo cual, da las bases para la constitución de la Responsabilidad Social en este contexto.

En el desarrollo del trabajo, se evidencian los condicionantes históricos que el modo de producción capitalista coloca para el surgimiento de la Responsabilidad Social, aquí se evidencia como el sistema de producción capitalista se reestructura para continuar con su reproducción y como éste envuelve otros sectores de la sociedad como el Estado y las organizaciones de la sociedad civil, modificándose según sus intereses.

Se analizó la reestructuración del sistema capitalista a partir de sus crisis y como estas determinaron el papel de los otros sectores sociales; esto es importante para comprender la base histórica que promueve la Responsabilidad Social como estrategia de su reproducción. 


\section{La responsabilidad social en el capitalismo contemporáneo}

Durante la crisis del sistema de producción en 1929, el capitalismo identificó que para mantener el orden era necesaria la intervención de una instancia como el Estado, con el fin de colaborar en la garantía de acumulación. El Estado absorbió en ese momento varias funciones que anteriormente no ejercía, como por ejemplo, la de mantenedora de la fuerza de trabajo debido a la necesidad de reproducir socialmente la clase trabajadora para que fuera consumidora en masa y así activar nuevamente la economía.

Posteriormente, en la década de 1960, según Mandel (1968), el patrón de acumulación fordista-keynesiano comienza a agotarse generando así otra crisis del capitalismo. Mota (1985) coloca algunas situaciones que demuestran esto; como la caída de las tasas de lucro, las variaciones en la productividad, el endeudamiento internacional y el desempleo. Ante este panorama, gana cuerpo la visión del Estado mínimo tanto en la producción de bienes y servicios, como en la regulación de las acciones del mercado.

Antunes (2001) indica que a partir de esa crisis, generada por un colapso en la superproducción efectuado por el régimen fordista, era necesario, para la reproducción del sistema de producción, implementar una nueva práctica de acumulación del capital, en ese contexto emergen nuevas formas organizacionales y de reajustamiento social y político, o sea, la llamada reestructuración productiva, la cual trajo cambios importantes, no solamente en la parte económica de los países, también genera cambios en la esfera política y social, afectando principalmente las condiciones de vida de la clase trabajadora debido a las transformaciones del Estado ante estas crisis.

Es de esa forma, que la profunda crisis del capital da sus primeras señales, articulada a la reestructuración productiva, se trata de crear un nuevo modelo que garanta el orden burgués; presentándose así el régimen de acumulación flexible. Harvey (1993) menciona que esta acumulación flexible consiste en la flexibilización de los procesos y de los mercados de trabajo además de los productos y patrones de consumo, el sentido de este nuevo sistema de acumulación es de producir el máximo con un mínimo de trabajadores (generando una explotación máxima de la fuerza de trabajo) integrando además nuevas tecnologías y técnicas gerenciales.

Según Mészáros (1995, como se cita en Antunes, 1999, p. 19), la explotación más intensa del trabajo se da por la falacia de la calidad total, que se puede traducir en tasa de utilización decreciente del valor de uso de las mercancías, que significa la disminución del tiempo entre producción y consumo, con reducción de la vida útil de los productos. Ese ritmo acelerado impone la innovación de los productos y de los procesos productivos, que se desenvuelven a partir de la introducción de nuevas tecnologías y nuevas formas de organización del trabajo, como la flexibilización del proceso productivo, la tercerización y diferentes formas de precarización de las relaciones laborales como trabajador part-time, subcontratado, la horizontalización de la empresa, el trabajo en equipo y de los círculos de control de calidad.

En este contexto de innovaciones tecnológicas, organización de la producción basada en la flexibilización, precarización del trabajo y disminución del tiempo de vida de las mercancías, llevan, según Antunes $(1999$, p. 53) a "um novo patamar de intensificação do trabalho, combinando fortemente as formas relativas e absolutas da extração de mais-valia".

Es necesario resaltar que el conjunto de elementos pertinentes a la reestructuración productiva aquí indicada conforma el ejemplo paradigmático del modelo de producción que se instauró en ese contexto: el toyotismo. La adaptación del modelo por otros países se subordinó a las particularidades de la región latinoamericana, tanto en los aspectos políticos, económicos culturales, sociales y geográficos, además como su relación con la economía mundial.

Esos hechos encima relacionados derivan de varios factores:

- Primero de la necesidad del capital de restaurar su producción, que modificó el patrón de producción en algunos sectores como por ejemplo el de servicios.

- Segundo, fue fundamental prevalecer el sector financiero, para la especulación, en detrimento del sector productivo, reflejo de la propia crisis del capital.

- Tercero, con la crisis del mecanismo de regulación del sistema, el Welfare State, identificada por los neoliberales, como crisis fiscal del Estado burgués, ocasionó la privatización de los servicios públicos, pues hubo la necesidad de disminuir los gastos públicos sociales, para mantener la acumulación, acarreando consecuencias nefastas para la clase trabajadora.

En este contexto, una vez más, el Estado utilizado como mecanismo para la reproducción del sistema de producción y para superar la crisis mencionada, ejecuta un conjunto de políticas macroeconómicas inculcadas por los Organismos Internacionales como Banco Mundial y el Fondo Monetario Internacional (FMI) en los países dependientes, las cuales nacen en los años 1980 a partir del conocido Consenso de Washington ${ }^{3}$; este conjunto de políticas son llamadas como Programa de Ajuste Estructural, los cuales sientan las bases para el neoliberalismo en América Latina ${ }^{4}$. 
Según Mota (1985, p. 30) este Consenso "deve ser pensado como um dos meios pelos quais a burguesia internacional imprime uma direção política de classe às estratégias de enfrentamento da crise dos oitenta, especialmente no que diz respeito às reformas a serem implementadas pelos países periféricos".

En consecuencia, como señala Paulo Netto (2003), en las últimas décadas las políticas económicas de los llamados ajustes en el mundo entero, han incrementado y acentuado los procesos de precarización de las condiciones de vida de la clase que vive del trabajo, como una de las etapas del capitalismo, el neoliberalismo expone dentro de la esfera económica una prominencia de las condiciones de vida de la clase que vive del trabajo, como una de las etapas del capitalismo, el neoliberalismo expone una preferencia absoluta del mercado y el libre juego de fuerzas de este.

Los puntos centrales de esa agenda era disminuir el déficit fiscal atribuido al gasto público (sobretodo el gasto social); promover la reforma del Estado, remodelando sus funciones en la perspectiva de aumentar su eficiencia a través de la disminución de costos; aumentar la competitividad reduciendo los costos sociales de las empresas y flexibilizando la mano de obra.

Sin embargo, la crisis es explicada, por los defensores de la reforma del Estado, como una crisis de la burocracia estatal de la administración pública, o sea, exceso de regulación, rigidez burocrática, privatismo e ineficiencia del servicio público, a partir de una incapacidad financiera y administrativa de gobernar. Luego, presenta la necesidad de garantizar condiciones de más gobernabilidad y gobernanza, más eficiencia para atender las demandas de los ciudadanos con mejor cualidad y en un costo menor (Bresser Pereira, 1998).

En ese sentido, se percibe un momento histórico de

\section{El ideal neoliberal cumple su}

función político-ideológica retirando la dimensión de derecho universal de

ciudadanía en relación a las

políticas sociales estatales, creando una cultura de autorresponsabilidad y de autoayuda para el enfrentamiento de las refracciones de la cuestión social. una reversión conservadora, en que la égida neoliberal propone una redefinición del papel del Estado, una reforma, que transfiere para el sector privado actividades que puedan ser controladas por el mercado, como, por ejemplo, menciona Behring (2003), por medio de las empresas estatales y las políticas sociales comercializables; otra forma es la descentralización para el sector público no estatal, de servicios que no envuelven el ejercicio del poder del Estado, más deben ser subsidiados por él. Se trata de la producción de servicios competitivos o no exclusivos del Estado, estableciendo convenios con la sociedad civil para el financiamiento y control social de su ejecución.

Es una nueva estrategia de legitimación del sistema capitalista, que implica en una serie de acciones direccionadas para el traslado de la lógica del Estado para las lógicas de la sociedad civil y el mercado (Montaño, 2003).

Es decir, los procesos de reestructuración del capital mencionados anteriormente, orientados por los principios neoliberales trajeron consigo, según Montaño (2003), una flexibilización de los mercados nacional e internacional, de las relaciones laborales, de la producción, de la inversión financiera, del distanciamiento del Estado de sus responsabilidades sociales y de la regulación social entre capital y trabajo, permaneciendo, sin embargo, como instrumento de consolidación hegemónica del capital mediante su papel central en el proceso de desregularización y reforma estatal y en la reestructuración productiva.

En este sentido, a partir de los hechos mencionados, el Estado retrocede en su actuación en la intervención social; en lugar de constituir una red universal de protección social que expanda el Estado en la garantía de los derechos sociales, el bienestar social recae en otro ámbitos privados; como menciona Montaño (2003), en las familias, en la comunidad, en las instituciones religiosas y filantrópicas, las cuáles deben de responsabilizarse por eso, en una supuesta red de solidaridad que proteja a los más pobres.

A partir de esa refuncionalización del Estado, se constata la legitimidad de otras fuentes para la ejecución de las políticas sociales, como por ejemplo la reglamentación del tercer sector, el cuál a través de una asociación entre Estado, ONGs y entidades filantrópicas, combinado con el servicio voluntario, se desprofesionaliza y despolitiza la intervención en el área social, remetiéndola al mundo de la solidaridad mutua, de la realización del bien común, por intermedio muchas veces de un trabajo voluntario no remunerado. Se trata de un verdadero retroceso histórico en relación a los derechos sociales conquistados por la clase trabajadora.

Montaño (2003) muestra como la racionalidad burguesa fragmenta la realidad y no la analiza en su totalidad, para que así se tengan respuestas fragmentadas e individuales a la cuestión social. Coutinho 
(2003) resalta que en momentos de crisis, la burguesía exacerba ideológicamente la irracionalidad subjetiva y que tanto el irracionalismo cuanto el racionalismo formal son expresiones imprescindibles del pensamiento ideológico de la burguesía contemporánea, inhábil de aceptar la razón dialéctica y la dimensión histórica de la objetividad.

Para esta estrategia neoliberal, primeramente se opera una separación y fragmentación radical (del agrado de la segmentadora visión positivista y la llamada posmodernidad) entre las cuestiones políticas, supuestamente reducidas al aparato estatal, en segundo lugar, según Montaño (2003), el neoliberalismo promueve el paso de las cuestiones económicas del Estado para el mercado y las cuestiones sociales para el tercer sector, manteniendo en el espacio estatal los procesos políticos formales y algún nivel de intervención social.

En el camino del pensamiento burgués neoliberal, existe una nueva estructuración social, en la cual el ciudadano de derechos se torna ciudadano cliente, consumidor de servicios de organizaciones, cuyo comportamiento se pauta por una perspectiva empresarial. Afirmar la necesidad de privatizar y minimizar las atribuciones del Estado, de forma que "os cidadãos e as organizações privadas devem assumir a responsabilidade pessoal pelo seu bem-estar e pelo futuro da sociedade" (Rockefeller, 1995, p. 25).

El mismo autor justifica que para la construcción de un país democrático y próspero es necesario el desarrollo de un "mecanismo estabilizador de um sistema democrático, uma terceira maneira de expressar e satisfazer necessidades, de agir e de alcançar objetivos, sem ter que enfrentar a rigidez e as ineficiências da burocracia governamental" (Rockefeller, 1995, p. 25). O sea, Rockefeller (1995) afirma la necesidad de fortalecer un tercer sector, que asuma la responsabilidad del Estado en el trato de las secuelas de la cuestión social, pues esa posibilidad, según él, más allá de ser la base financiera indispensable a la filantropía (Rockefeller, 1995, p. 25), parte también de la iniciativa personal y de la articulación de un número incontable de personas, grupos y organizaciones comunitarias, cuyos objetivos son los de reformar la sociedad y abordar cuestiones que afecten la calidad de vida.

Tenemos clareza de que el proyecto neoliberal, evidenciado en este discurso anteriormente expuesto, tiene como objetivo desreglamentar los mercados y reformar el Estado, privatizando sectores estratégicos de la economía, más allá de la retirada sistemática de derechos y garantías de la clase trabajadora.

Sabemos pues,

o que o grande burguês e seus executivos desejam e pretendem, em face da crise contemporânea da ordem do capital, é erradicar mecanismos reguladores que contenham qualquer componente democrático de controle do movimento do capital. O que desejam e pretendem não é "reduzir a intervenção do Estado", mas encontrar as condições ótimas (hoje só possíveis com o estreitamento das instituições democráticas) para direcioná-la segundo seus particulares interesses de classe. (Paulo Netto, 1995, p. 44)

El ideal neoliberal cumple su función político-ideológica retirando la dimensión de derecho universal de ciudadanía en relación a las políticas sociales estatales, creando una cultura de autorresponsabilidad y de autoayuda para el enfrentamiento de las refracciones de la cuestión social. Generando, a partir de la precarización y de la focalización de la intervención estatal, una demanda altamente lucrativa para el sector empresarial, sea por la mercantilización de esos servicios o a través del marketing social que es promovido por la Responsabilidad Social.

En América Latina, después de los cambios que trajo los Programas de Ajuste Estructural en los años 1980 y 1990, modificó la intervención del Estado en lo social, por ejemplo, los servicios que antes eran públicos pasaron a ser privados, las economías se abrieron a la competencia internacional, las mujeres ingresaron masivamente al mercado de trabajo, además, la distribución de la riqueza y con los ingresos han seguido polarizándose, son millones las personas que viven en condición de pobreza y las que deben migrar de sus lugares de origen para conseguir empleos y generar ingresos.

A pesar de las particularidades que han marcado los diferentes países de América Latina, estas características son generales, más allá de sus especificidades nacionales al ser una región tan heterogénea, en palabra de Paulo Netto (2016, p. 64) "o subcontinente latino-americano, do México à Patagônia Argentina, sob todos os aspectos, é um universo extremadamente diferenciado".

En Centroamérica, específicamente en Costa Rica, Guendell (1990) indica que ha sido un país diferenciado de los demás países de la región ya que logra crear un Estado ampliado en la década de los 1950, posibilitando un desarrollo relativamente sostenible en la actividad estatal. Como refiere el mismo autor, la crisis económica reduce la capacidad de financiamiento de las políticas sociales y al mismo tiempo provocó un empobrecimiento mayor en la población y por consiguiente, un incremento en los movimiento y demandas sociales.

Por su parte, la crisis política en El Salvador y Guatemala, obligó al Estado a subordinar algunas de las políticas sociales a criterios de carácter político-militares, en el plano administrativo, la crisis exacerbó el carácter irracional de las políticas sociales y mostró la reducida eficacia de los programas sociales. 
Por otra parte, para comienzos de los años 1980, los Estados de América Central, menos Nicaragua, fomentaron como consecuencia de la crisis, políticas de estabilización económica de corte neoliberal basadas en acuerdos con el FMI. Las nuevas políticas, según Guendell (1990), establecieron nuevos límites al gasto social y accionaron un proceso de racionalización de la actividad pública que se direccionaba a las prioridades de intervención en el campo económico.

Posteriormente, se evolucionó para una reestructuración gradual, durante esta segunda fase que se inicia a partir de 1983. Se atenuaron los efectos de la crisis económica sobre la política social, a raíz de la asistencia económica de Estados Unidos y la ejecución de políticas económicas de naturaleza selectiva.

Con la crisis económica, las instituciones de bienestar y asistencia social, que funcionaban con una lógica sustentada en los resultados más que en la satisfacción de metas y basaban por tanto su gestión en modelos y procedimientos políticos-administrativos que priorizaban la satisfacción de intereses económicos y políticos particulares, vieron disminuidos sus recursos financieros.

Además, la crisis deterioró las condiciones de vida de las personas de la clase trabajadora, incrementando la demanda de servicios estatales, principalmente en el campo de salud y subsidios familiares. Es así que la intervención en las políticas sociales en la actuación en lo social por parte de diferentes actores se torna importante, no por iniciativas individuales de grupos, empresas u organizaciones sociales, si no por la intencionalidad del sistema de producción que dirige de acuerdo a sus intereses.

Como menciona Pereira (2004), para los neoliberales, el bienestar está asociado al mérito individual y no a los derechos de la ciudadanía social, a la productividad capitalista libre de controles y no a la redistribución de bienes y servicios colectivos y a la igualdad de oportunidades y no la igualdad de resultados.

En esta lógica, entra el papel primordial de las empresas en su intervención en las políticas sociales a pesar de que el interés radica en resguardar su imagen y reputación de conductas irresponsables en el campo social y ambiental, lo que interfiere directamente en su productividad.

Según Prado, Flores, Pratt y Ogliastri (2004), experiencias de compañías multinacionales y de pequeñas y medianas empresas han demostrado que desarrollar prácticas de Responsabilidad Social genera beneficios tangibles en las diferentes áreas de operación del negocio. Diferentes organismos internacionales como el Banco Mundial, la Organización de las Naciones Unidas (ONU) o World Business Council for Sustainable Development (WBCSD) y otros organismos promotores de la Responsabilidad Social, destacan beneficios importantes por la adopción de prácticas de este tipo.

Por otra parte, otro de los sectores que han tenido un fuerte auge a partir de los años 1970 son las organizaciones comunales, donde una de las observaciones más importantes que expone el discurso conservador dicho por los neoliberales sobre estas organizaciones es lo que se refiere a la conveniencia, capacidad y consentimiento de este sector para ser el responsable por la carga extra, o sea, por programas de índole social que anteriormente eran responsabilidad del Estado.

Así, la Responsabilidad Social tanto de las empresas como de las organizaciones comunales toma importancia, ya que se desarrolla en un contexto impulsado por una serie de factores económicos y políticos presentados anteriormente, promovido por un discurso de responsabilidades compartidas y disminuyendo las responsabilidades del Estado de su intervención en lo social, lo cual, no se limita solamente para definir quién interviene, sino que traspasa la cantidad y calidad de las políticas sociales existentes, donde cada vez son más restringidas y focalizadas a la población.

\section{Conclusión}

Considerando los datos presentados, es notable que haya existido no solamente una regresión de los derechos sociales a partir de la llamada reforma del Estado, especialmente en los países de América Latina, sino un cambio en su direccionalidad, teniendo por objetivo la despolitización de la contradicción capital/trabajo y su consecuente desigual distribución de la riqueza social, retomando la idea a favor en el trato a la cuestión social considerándola en el ámbito inmediato e individual.

En Brasil, por ejemplo, esto se dio con el Plano Diretor da Reforma do Aparato do Estado, elaborado por el Ministério da Administração Federal e Reforma do Estado (MARE) en 1995, que conformó los postulados del Consenso de Washington, con el fin de "reformar" el Estado de su forma a substituir la administración burocrática, por un nuevo modo de administración pública de tipo gerencial, "modernizar el Estado" y conformarlos a los moldes empresariales.

Igualmente en los países centroamericanos, la misma situación se dio con los Planes de Ajuste Estructural, en Costa Rica por ejemplo, esto se profundiza en 1995 con el tercer Plan, donde caló en las políticas sociales del país y por ende en las condiciones de vida de la población. 
Esta reversión conservadora se ejemplifica en la refilantropización de la cuestión social, con la actuación de la Responsabilidad Social de las empresas, del llamado tercer sector y del propio Estado, que se coloca en este discurso como un ente fiscalizador.

Lo importante de considerar la trascendencia de estos préstamos internacionales que se materializa con los Planes de Ajuste Estructural, radica en que no solamente constituye una forma de impregnar los intereses de los Organismos Internacionales (que representan los intereses del capital) en los países considerados como dependiente, también, constituyen una forma de neocolonización, delimitando y organizando la división social del trabajo internacional para la reproducción del sistema de producción capitalista.

Así, no podemos olvidar que esa idea de la Responsabilidad Social, como forma de beneficiar el interés de todos, tiene un supuesto consenso a partir de un pacto social de colaboracionismo entre las clases, en otros términos, una suposición de que no existe más antagonismo entre las clases, apenas diferencias entre ellas.

Todo ese proceso integra una amplia reestructuración del capital que tiene como uno de sus objetivos fundamentales la implementación de la reforma del Estado que busca regular la relación entre Estado y sociedad civil para conducir una nueva sociabilidad en la construcción de un nuevo consenso para tornar hegemónica esa nueva cultura de un pacto social.

En este camino, las manifestaciones de la cuestión social son interpretadas como incapacidad individual y debe ser enfrentada por medio de valores morales positivos universales y mecanismos relacionados a la ayuda mutua, en ese escenario se posiciona el supuesto ideal de un capitalismo responsable o sostenible, reformado y ético moralmente.

Se pretende, a partir de esta lógica neoliberal, responder a las demandas de la cuestión social a partir de una movilización social de la comunidad local, ONGs, empresas privadas, por medio de la llamada articulación con el Estado y se reconocen las empresas ciudadanas como nuevos agentes de promoción social, sin embargo, olvidan de explicar cómo serán prestados esos servicios sociales - vía mercado u ofertados por el trabajo voluntario - que muchas veces convocan personas no cualificadas para tales actividades. Esto no es, para ellos, política asistencialista o paternalista, más si una supuesta democracia plena.

De esta manera, la función social de la respuesta a la cuestión social deja de ser, en este proyecto neoliberal, responsabilidad privilegiada del Estado y por medio de este conjunto de la sociedad y pasa a ser ahora de autorresponsabilidad de los propios sujetos de necesidades y de la acción filantrópica.

La Responsabilidad Social va de la mano con el discurso del capitalismo consciente, el cuál manifiesta la falacia de invitar a las empresas a reflexionar sobre la sociedad y su impacto, sin embargo, este tipo de discursos, lo que genera es una falsa conciencia en la población por el cuál este tipo de programas, aún, fomentadas por los gobierno, son formas de despolitizar las políticas sociales. Un ejemplo de esto, es el caso guatemalteco, donde algunas empresas han utilizado programas de Responsabilidad Social para resolver problemas con las comunidades, especialmente en comunidades indígenas donde la inversión privada va en contra de su cultura, forma de vivir y planes territoriales.

Este discurso de sostenibilidad es legitimado también por el discurso posmoderno, el cual constituye la base cultural del capitalismo contemporáneo, que lleva a esta despolitización. Se afirma la tesis del fin de la sociedad del trabajo, el fin de la historia, es decir, que no existen posibilidades más allá del capitalismo, sin embargo, nada es más contradictorio en afirmar el fin de las utopías y pregonar la posibilidad de un capitalismo sostenible ya que la base de éste es la desigualdad y explotación.

\section{Referencias}

Anderson, P. (1995). Balanço do neoliberalismo. In E. Sader \& P. Gentili (Orgs.), Pós-neoliberalismo: As políticas sociais e o Estado democrático (pp. 9-23). Rio de Janeiro, Brasil: Paz e Terra.

Antunes, R. (1999). Os sentidos do trabalho: Ensaio sobre a afirmação e a negação do trabalho. São Paulo, Brasil: Boitempo.

Antunes, R. (2001). ¿Adiós al trabajo? Ensayo sobre las metamorfosis y la centralidad del mundo del trabajo. São Paulo, Brasil: Cortez. Behring, E. R. (2003). Brasil em contra-reforma: Desestruturação do Estado e perda de direitos. São Paulo, Brasil: Cortez.

Bresser Pereira, L. C. (1998). Reforma do Estado para a cidadania: A reforma gerencial brasileira na perspectiva internacional. São Paulo, Brasil: 34.

Coutinho, J. (2003). Desmistificando o “terceiro setor”. Lutas Sociais, 2003(9/10), 183-185. Recuperado de https://revistas.pucsp.br/ ls/article/view/25783/18427

Guendell, L. (1990). La política social en Centroamérica en los ochenta: Crisis y reestructuración. Recuperado de http://www.ts.ucr.ac.cr/ binarios/docente/pd-000134.pdf

Harvey, D. (1993). Condição pós-moderna: Uma pesquisa sobre as origens da mudança cultural. São Paulo, Brasil: Loyola. 
Iamamoto, M. (2011). Serviço Social em tempo de capital fetiche: Capital financeiro, trabalho e questão social (5. ${ }^{\text {a }}$ ed.). São Paulo, Brasil: Cortez.

Mandel, E. (1968). Formación del pensamiento económico de Marx: De 1843 a la redacción de El Capital. México, D.F., México: Siglo Veintiuno.

Ministério da Administração Federal e Reforma do Estado. (1995). Plano diretor da reforma do aparelho do Estado. Recuperado de http://www.biblioteca.presidencia.gov.br/publicacoes-oficiais/catalogo/fhc/plano-diretor-da-reforma-do-aparelho-do-estado-1995.pdf Montaño, C. (2003). Terceiro setor e questão social: Crítica ao padrão emergente de intervenção social (2. ${ }^{\text {a }}$ ed.). São Paulo, Brasil: Cortez. Mota, A. (1985). O feitiço da ajuda: As determinações do Serviço Social na empresa. São Paulo, Brasil: Cortez.

Paulo Netto, J. (1995). Crise do socialismo e ofensiva neoliberal (2. ed.). São Paulo, Brasil: Cortez.

Paulo Netto, J. (2003). Cinco notas a propósito de la "cuestión social”. In E. Borgianni, Y. Guerra, \& C. Montaño (Orgs.), Servicio Social crítico. Hacia la construcción del nuevo proyecto ético-político profesional (pp. 43-55). São Paulo, Brasil: Cortez.

Paulo Netto, J. (2016). Tendências do Serviço Social na América Latina e no Brasil. In A. Martins, C. M. dos Santos, D. S. Simões, J. Ferreira, \& M. Braz (Orgs.), Serviço Social Portugal-Brasil: Formação e exercício em tempos de crise (pp. 63-71). Campinas, Brasil: Papel Social.

Pereira, P. A. P. (2004). Pluralismo de bem-estar ou configuração plural da política social sob o neoliberalismo. In I. Boschetti, P. A. P. Pereira, M. A. César, \& D. B. B. de Carvalho (Orgs.), Politica social: Alternativas ao neoliberalismo (pp. 135-159). Brasília, Brasil: Editora Universidade de Brasília.

Prado, A., Flores, J., Pratt, L., \& Ogliastri, E. (2004). Marco lógico y conceptual del modelo de responsabilidad social empresarial para Costa Rica. Recuperado de https://www.researchgate.net/publication/264846671_Marco_Logico_y_Conceptual_del_Modelo_ de_Responsabilidad_Social_Empresarial_para_Costa_Rica

Rockefeller, D. (1995). A filantropia e o futuro do Brasil. In Seminário internacional, cidadania participativa: Responsabilidade social e cultural num Brasil democrático (pp. 17-26). Rio de Janeiro, Brasil: Texto e Arte, 1995.

\section{Notas}

1 Parafraseando a Anderson (1995), los países que adoptaron el recetario neoliberal se dedicaron a elevar las tasas de interés, reducir los impuestos de los más ricos, crear niveles masivos de desempleo, combatir fuertemente el poder de los sindicatos, cortar drásticamente los gastos sociales e implementar un amplio programa de privatizaciones, principalmente de las industrias de petróleo, acero, electricidad y agua.

2 Iamamoto (2011, p. 124) resalta que "na raiz do atual perfil assumido pela questão social encontram-se as políticas governamentais favorecedoras da esfera financeira e do grande capital produtivo." Paulo Netto (2003) al respecto menciona que resultaron de un cuadro de radicalización de la cuestión social, ampliando antiguas y recreando nuevas manifestaciones.

3 Se destaca el denominado Consenso de Washington, que refiere al conjunto de políticas económicas y sociales de corte neoliberal, aplicadas a partir de los años 1980 para enfrentar la crisis económica, estas medidas, llamadas de ajuste estructural, eran condicionadas por el financiamiento concedido por estos Organismos a países llamados dependientes.

4 Dentro de las orientaciones presentes en el Consenso, se destaca la desestructuración de los sistemas de protección social vinculados a las estructuras estatales y la privatización de los mismos. De esa forma, las políticas sociales en la década de 1990 ya son direccionadas para el combate de la pobreza y para dividir la responsabilidad entre Estado y sociedad en el enfrentamiento de la cuestión social.

\section{Mariangel Sánchez Alvarado}

mariangelsa@gmail.com

Máster en Política Social por la Universidade Federal de Mato Grosso (UFMT)

Doctoranda en Trabajo Social por la Universidade Federal de Alagoas (UFAL)

\section{UFAL}

Av. Lourival Melo Mota, s/n - Tabuleiro do Martins

Maceió - Alagoas - Brasil

CEP: $57.072-970$

\section{Tânia Maria Santana dos Santos}

taniamss@terra.com.br

Doctorado en Trabajo Social por la Pontifícia Universidade Católica de São Paulo (PUC-SP)

Docente del Departamento de Trabajo Social y del Programa de Posgrado en Política Social de la Universidade Federal de Mato Grosso (UFMT) 


\section{UFMT}

Av. Fernando Correa da Costa, s/n - Coxipó

Cuiabá - Mato Grosso - Brasil

CEP: 78.000-000

\section{Agradecimientos}

Agradecemos ao Programa de Pós-Graduação em Política Social da UFMT, assim como ao programa PAEC-OEA.

\section{Agencia financiadora}

No se aplica.

\section{Contribuciones de las autoras}

$\mathrm{O}$ artigo foi realizado a partir da dissertação intitulada "A Responsabilidade Social na Costa Rica" do Programa de Mestrado em Política Social da UFMT, sob a orientação da Prof. Tânia Santana. A autora Mariangel Sánchez realizou a revisão bibliográfica e documental, a análise da informação e o texto do artigo.
A autora Tânia Santana realizou aportes nas discussões, considerações finais e as correções pertinentes.

Aprobación por Comisión de Ética y consentimiento para participación

No se aplica.

Consentimiento para publicación

No se aplica.

\section{Conflicto de intereses}

No hay conflicto de intereses. 